\title{
Intentional learning Vs incidental learning
}

\begin{abstract}
This study is conducted to demonstrate the knowledge of intentional learning and incidental learning. Hypothesis of this experiment is intentional learning is better than incidental learning, participants were demonstrated and were asked to learn the 10 non sense syllables in a specific sequence from the colored cards in the end they were asked to recall the background color of each card instead of non-sense syllables. Independent variables of the experiment are the colored cards containing non-sense syllables which are to be memorized by the participant; dependent variables are the number of correct response made by the participant. The findings of the experiment concluded that intentional learning is better than incidental learning, hence hypothesis is proved.
\end{abstract}

Keywords: intentional learning, incidental learning, non-sense syllable cards (colored and uncolored), score-sheet
Volume 7 Issue 2 - 2017

\author{
Shahbaz Ahmed \\ Karachi Medical and Dental College, Pakistan
}

Correspondence: Shahbaz Ahmed, Karachi Medical and Dental College, Pakistan,Email dr.shehbaz2012@yahoo.com

Received: May 08, 2016 | Published: January 17, 2017

\section{Introduction}

This study was conducted to show that intentional learning is better than incidental learning and to highlight the idea that incidental learning an automatic existence.

Learning is the attainment of knowledge or skills that we gain via experiences, studies or when we are taught. There are many different types of learning in psychology but the ones the relevant ones to be discussed in detail are incidental learning and accidental learning.

Incidental learning is the learning of one stimulus feature while concentrating on another stimulus feature too. A little more specific connotation of incidental learning is that it involves the learning of formal aspects through a center of attention on semantic aspects. Incidental learning can occur in many modes inclusive of observations, communications with colleagues about tasks or projects, experiencing mistakes, assumptions and adapting to new situations. A reactive component of incidental learning occurs in the middle of a task completion action when there is little time to think. Incidental learning has also been portrayed as implicit when knowledge is acquired independent of conscious attempts to learn. ${ }^{1}$ A little broader meaning of incidental learning is taken in a pessimistic way as it is the learning without the intent to learn.

Whereas, intentional learning is described as the having the intention to learn the material and to commit it to ones memory. It explains the study conditions where participants are forewarned that they will be tested on material to which they are exposed. Participants in an intentional learning task are told in advance that they will be tested in after the learning phase; they will try to store the word information that is to be learned in a form perceived as a transferable to the test situation. And processing instructions during the learning phase in an incidental learning setting may or may not be conducive to successful transfer to the test situation. ${ }^{2}$

Although learning in everyday life is both incidental and intentional but intentional learning instructions produce better recall and recognition performance than incidental learning instructions. Incidental and intentional learning refers, strictly speaking, only to absence or presence of an announcement to participants in a psychological experiment as to whether they will be tested after the experiment task. ${ }^{2}$

Through incidental learning, inferring the meaning of the word, however this process offers no guarantee for the retention of the link between the word's form and its meaning. In other words guessing from the context does not necessarily result in long-term retention. This finding verifies the study of Parry (1993), Mondria and Wit (1991). As a result even if they happen to acquire a word incidentally from reading passage, it is likely that they will be quickly forgotten after a week.

Another factor which plays an important role is method of Anticipation. It is defined as an experiment paradigm in learnt and memory research in which a timid sequence of stimuli (like a list of words) is proposed to the subject to be memorized. After a gap the sequence is represented and the subject is requested to guess the following stimuli at each stage of the sequence so that each stimulus is a cue for the following response. In an anticipation procedure item is first presented while subject attempts to give the associated response, and is followed immediately by the presentation of the correct response, usually paired with its stimulus. ${ }^{3}$

Trial and Error models encourage a useful exploratory side of learning. Trial and Error learning is a fundamental method of solving, it is characterized by repeated, varied attempts which are continued until success or until the agent stop trying. Thorndike performed an experiment in lab in which an animal comes to associate particular behaviors with the consequences they produce. This tends to reinforce the behavior. Evidence from lower species and even infants primates suggests that learning may occur through the repeated pairing (under conditions of reinforcement) of particular stimulus and response combinations. ${ }^{4}$

In a problem solving task, the agent is repeatedly presented with instances of the task (a series of trials). In each trial the agent is presented with an instance of the problem to be solved (i.e., an initial state). The agent's objective is to execute a sequence of actions that drives the world into a desirable goal state. When the goal is achieved the agent receives a positive reward and the trail ends. If after a predetermined number of steps the agent fails to solve a problem, it gives up and goes on to the next trial.

It is presumed that awareness of certain thing gives the participants the option to bring an alternative active strategy for acquiring the sequential knowledge, just as in trial and error learning. Two hypotheses exist to explore incidental and intentional learning.

1. Intentional learning is better than incidental learning. 
2. If the subject is able to recall the colors of the cards then it is proved that incidental learning exists. Variables of experiment are as follows:

3. Independent variable is the cards containing words and colors which are to be memorized by the subject.

4. Dependent variable is the number of correct responses (words and color guessing) the subject makes.

\section{Sample}

The sample of the experiment was a group of 20 undergraduate students studying at the Institute of Business Administration. The number of male and female participants was equal.

\section{Measures used in this experiment are}

a. Non-sense syllable cards (colored and uncolored)

b. Score-sheet

c. Stop watch

d. Pencil/pens

\section{Procedure}

To initiate the experiment several participants were approached. They were asked to stay in the class room after the class. The experiment was conducted individually on each participant. They were recruited and were asked to get settled on their chair in front of the experimenter. After reading aloud the instructions in front of each participant individually the experimenter was certain that subject has understood the procedure.

The experiment consisted of two phases. Phase 1 was designed to check the participant's intentional learning. Participant was shown ten different cards each of a different color and a unique non-sense syllable. Each card was shown for 20 seconds. Participant was asked to memorize the words in the exact same sequence, and the experimenter made sure to inform the participant that s/he will later be tested on his/ her memorization. The response was recorded in the following chart.

\begin{tabular}{lllllllllll}
\hline & I & $\mathbf{2}$ & $\mathbf{3}$ & $\mathbf{4}$ & $\mathbf{5}$ & $\mathbf{6}$ & $\mathbf{7}$ & $\mathbf{8}$ & $\mathbf{9}$ & $\mathbf{1 0}$ \\
RIV & & & & & & & & & \\
MUP & & & & & & & & & & \\
DAJ & & & & & & & & & & \\
ZOL & & & & & & & & & & \\
VOF & & & & & & & & & & \\
CAH & & & & & & & & & \\
YEB & & & & & & & & & & \\
WEJ & & & & & & & & & & \\
FIH & & & & & & & & & & \\
BUJ & & & & & & & & & & \\
\hline
\end{tabular}

Participants were given several attempts, and the process was repeated until they got all the words correct or they stopped trying. Tick/cross marks were made in the given chart to record whether the word was memorized or not. The words written in the first column represent the words written on the cards respectively.

Once all the words were memorized by the participant, s/he qualified for phase 2 which was designed to check the participant's incidental learning. In this phase, participant was shown the same ten cards, but with a twist. These cards were white. Now the participant was asked to recall the color of the card containing the same syllables. The syllables were shown in the exact same sequence. The results were recorded in the following chart.

\begin{tabular}{ll}
\hline Card & Color \\
RIV & \\
MUP \\
DAJ \\
ZOL \\
VOF \\
CAH \\
YEB \\
WEJ \\
FIH \\
BUJ
\end{tabular}

The color that the participant recalled was recorded in the "color" column. And later, the experimenter tallied the colors recalled by the participant with the original color of the card.

Finally, the results were critically analyzed to find out whether the results supported the hypotheses of the experiment or not.

\section{Results}

The results of this experiment support our hypotheses. Let us recall what the hypotheses were:

H1: Incidental Learning exists.

$\mathrm{H} 2$ : Intentional Learning is better retained than incidental learning.

A graphical representation of Phase 1 is as follows:

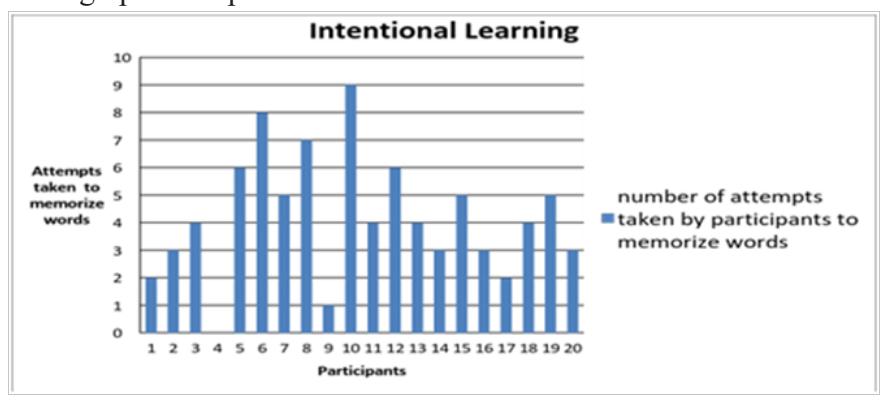

This graph suggests that all the participants were able to memorize all the syllables in 3-8 attempts. And now let us look at a graphical representation of Phase 2, which is as follows:

The graph suggests that most of the participants were not able to recall all the words. There was only the exception of one participant who was keen to recall all of them.



Now to proof our hypotheses, we cannot state anything without combining the results of both of the phases, so here is a graphical representation of both the phases:

This graph suggests that although majority of the participants memorized the words in more than 3 attempts, the non-sense syllables 
were better retained. The more attempts that were taken by the participants, the more words they had memorized. However, it is safe to say that Incidental learning also occurs while learning intentionally. After the participants had memorized all the words, they were asked to tell the colors that each word contained. Many participants struggled during this process as they had only memorized the non-sense syllables which they were asked to do. However, participants did manage to retain some of the colors incidentally. It was also noted that the nonsense syllables that participants most struggled for learning, the better they were at retaining the color of that particular word. There was one exception in our study in which a participant managed to retain all the colors incidentally but struggled a bit while memorizing the word.

Hence, through our study we were able to proof that incidental learn exists but intentional learning produces better recall as compared to incidental learning.

\section{Discussion}

Through this experiment, we deduced knowledge about incidental and intentional learning and successfully demonstrated that intentional learning is better than incidental learning. Overall result of this experiment supported the hypothesis we presented at the beginning. Intentional learning instructions produce better recall and recognition performance than incidental learning instructions. Participants in an intentional vocabulary learning task are told in advance that they will be tested after the learning phase, therefore, they try to store the word that is to be learned in a form perceived as transferable to the test situation, and if information is processed about a word during the learning phase, then there are fair chances of it being recalled for later use. The idea was first formalized as the Depth of the Processing Hypothesis.

Research on learning from context shows that incidental learning does occur unconsciously, but it has its limitations and prerequisites such as learning being small and cumulative. This is the reason why with some exceptions, most of the participants were able to recall few colors only. What matters is the motivational cognitive dimensions of the task, i.e. high will of involvement. Involvement in a word induced by the task will result in better retention means motivation for recalling the sequence correctly will engage the participants in more rehearsals of the sequence, hence more trials will result in higher number of correct responses and more colors being recalled.

This even accounts for the limited sample size of our experiment Only 20 subjects were able to be approached by the experimenter due to such a long and time-consuming procedure. One of the main problems that were faced was the unwilling attitude of the participants. Some subjects were not even willing to pursue the experiment after the first trial. ${ }^{5-7}$

Deception was compensated through de-briefing. Information was revealed about the true nature of the research to give them opportunities to discuss their feelings. Even at the initial stage, participants had the right to agree or refuse to participate in the research. Confidentiality was maintained, and thus, identities of all participants have been protected.

So we discussed the findings in context of research suggesting that incidental learning exists and intentional learning is better than incidental learning.

\section{Acknowledgments}

None.

\section{Conflicts of interest}

Author declares there are no conflicts of interest.

\section{Funding}

None.

\section{References}

1. Eraut M. The practice of reflection. Learning in Health and Social Care. 2004;3(2):47-52.

2. Catherine JD. The handbook of second language Acquisition. UK, Blackwell Publishing Ltd. 2003.

3. Batting FW, Brackett RH. Comparison of Anticipation and Recall Methods in Paired Association Learning. Psychological Report. 1961;9:59-65.

4. Brown RG, Redondo-Verge L, Chacon JR, et al. Dissociation between intentional and incidental sequence learning on Hungtinton's diseases. Brain. 2001; 124(Pt 11):2188-2202

5. Callander S. Searching and learning by Trial and Error. American Economics Review. 2011;101(6):1-2.

6. Steven DW, Dana HB. Learning to Perceive and Act by Trial and Error Machine Learning. 1991;7(1):45-83.

7. Mark AM, Michael EM. Effectiveness Process on Immediate and Delayed Recall. Program on Cognition Factors in Human Learning and Memory Report . 1976;51:1-21. 